\title{
REPRESENTAÇÕES SOCIAIS DA BIOSSEGURANÇA POR PROFISSIONAIS DE ENFERMAGEM DE UM SERVIÇO DE EMERGÊNCIA
}

\author{
Social Representations of Biosecurity by Nursing \\ Professionals at an Emergency Service \\ Representaciones Sociales de la Bioseguridad por \\ Profesionales de Enfermería de un Servicio de Emergencia
}

\author{
Andréia Rodrigues Moura da Costa Valle ${ }^{1}$ \\ Maria Eliete Batista Moura ${ }^{4}$
}

\author{
Mageany Barbosa Feitosa² \\ Ana Maria Ribeiro dos Santos ${ }^{5}$
}

\author{
Verônica Moura Diniz Araújo ${ }^{3}$ \\ Claudete Ferreira de Souza Monteiro ${ }^{6}$
}

\section{Resumo}

0 estudo objetivou apreender as Representações Sociais da Biossegurança elaboradas por profissionais de Enfermagem e analisar como essas representações influenciam na prática e na qualidade da assistência. Trata-se de uma pesquisa exploratória, com base na Teoria das Representações Sociais, realizada no serviço de emergência de um hospital público, com 60 profissionais de enfermagem. Os dados foram coletados através da técnica de Associação Livre de Palavras, utilizando as palavrasestímulos: biossegurança e infecção hospitalar, processadas no software Tri Deux Mots, e fazendo Análise Fatorial de Correspondência. Os resultados mostraram que a biossegurança foi objetivada através das evocações descartex, anti-sepsia e imunização, enquanto a infecção hospitalar foi objetivada através das evocações doença e sujeira. Considera-se, finalmente, que a biossegurança ainda não foi incorporada como um conjunto de medidas necessárias ao controle da infecção hospitalar, especialmente do ponto de vista social e psicológico, predominando os aspectos epidemiológicos, biológicos e econômicos.

Palavras-chave: Enfermagem. Exposição a Agentes Biológicos. Infecção Hospitalar. Psicologia Social.

\begin{abstract}
The objective of the study was to learn the Social Representations of Biosecurity elaborated by nursing professionals and to analyze how these representations influence the practice and quality of the assistance given. This is an exploratory study based on the Theory of Social Representations, and was done at an emergency service in a public hospital with 60 nursing professionals. The data was collected through the technique of Free Word Association by using the words as stimulus: biosecurity and hospital infection. The data was processed on the Tri Deux Mots software and a Correspondence Factor Analysis was done. The results showed that biosecurity was seen through the evocations disposable, anti-septic, and immunization, while hospital infection was seen through the evocations disease and uncleanness. Finally, it should be noted that Biosecurity has still not been incorporated as a group of measures necessary to control hospital infections. The economical, biological and epidemiological aspects predominate especially from the social and psychological point of view.
\end{abstract}

Keywords:Nursing. Exposure to biological agents. Cross Infection. Psychology Social.

\section{Resumen}

El estudio tiene como objetivo aprender las Representaciones Sociales de la Bioseguridad elaboradas por profesionales de enfermería y analizar como esas representaciones tienen influencia en la práctica y en la calidad de la asistencia. Se trata de una investigación exploratoria, con base en la Teoría de las Representaciones Sociales, fue realizada en el servicio de emergencia de un hospital público, con 60 profesionales de enfermería. Los datos fueron colectados a través de la técnica de Asociación Libre de Palabras, utilizando las palabras estímulos: Bioseguridad e infección hospitalaria, procesados en el software Tri Deux Motsy hecho Análisis Factorial de Correspondencia. Los resultados mostraron que la Bioseguridad fue objetivada a través de las evocaciones descartex, antisépsia e inmunización, mientras que la infección hospitalaria fue objetivada a través de las evocaciones, enfermedad y basura. Se considera finalmente, que la Bioseguridad aún no fue incorporada como un conjunto de medidas necesarias al control de la infección hospitalaria, especialmente del punto de vista social y psicológico, predominando los aspectos epidemiológicos, biológicos y económicos.

Palabras clave: Enfermería. Exposición a agentes biológicos. Infección Hospitalaria. Psicología Social.

'Enfermeira, Coordenadora da ESF do Município Aroazes - Piauí. Especialista em Saúde Coletiva e Saúde da Família. UFPI - Teresina. Enfermeira da ESF do Município Piripiri - Piaú. Especialista em Saúde Coletiva e Saúde da Família. UFPI - Teresina. ${ }^{3}$ Enfermeira. Especialista em Saúde Coletiva e Saúde da Família. UFPI - Teresina. ${ }^{4}$ Doutora em Enfermagem pela Escola de Enfermagem Anna Nery da UFRJ. Profa da Graduação e do Curso de Mestrado em Enfermagem da Universidade Federal do Piaú - UFPI. Coordenadora do Curso de Especialização em Prevenção e Controle das Infecções em Serviços de Saúde da Faculdade NOVAFAPI. Coordenadora de Pesquisa e PósGraduação da Faculdade NOVAFAPI. Presidente da ABEn - Seção Piauí. E-mail: posgraduacao@novafapi.com.br. ${ }^{5}$ Mestre em Enfermagem pela Escola de Enfermagem Anna Nery da UFRJ. Professora da Faculdade NOVAFAPI e da Universidade Federal do Piauí - UFPI. Membro da ABEn - Seção Piauí. ${ }^{6}$ Doutora em Enfermagem pela Escola de Enfermagem Anna Nery da UFRJ. Profa da Graduação e Coordenadora do Mestrado em Enfermagem da Universidade Federal do Piauí - UFPI. Profa da Faculdade NOVAFAPI. Membro da ABEn - Seção Piauí. 


\section{INTRODUÇÃO}

Na área da saúde, a biossegurança suscita reflexões por parte dos profissionais, especialmente dos que trabalham nas áreas críticas dos hospitais, uma vez que estão mais suscetíveis a contrair doenças advindas de acidentes de trabalho, através de procedimentos que envolvem riscos biológicos, químicos, físicos, ergonômicos e psicossociais.

0 risco biológico é o mais comum entre os profissionais de saúde. Esse risco aumentou após o surgimento da síndrome da imunodeficiência adquirida - AIDS e do crescimento do número de pessoas infectadas pelos vírus da hepatite $\mathrm{B}$ e $\mathrm{C}^{1}$.

É importante salientar que nos serviços de saúde, especialmente de urgência e emergência, grande parte dos acidentes que envolvem profissionais da área da saúde se deve à não-observância e obediência às normas de segurança. Contudo, o emprego de práticas seguras e 0 uso de equipamentos de proteção adequados reduzem significativamente o risco de acidente ocupacional, fazendo-se necessários, também, a conscientização dos profissionais para utilização de técnicas assépticas e o estabelecimento de normas de conduta e procedimentos que garantam ao profissional e ao paciente um tratamento sem risco de contaminação.

Dessa forma, Biossegurança é definida como:

Conjunto de ações voltadas para a prevenção, minimização ou eliminação de riscos inerentes às atividades de pesquisa, produção, ensino, desenvolvimento tecnológico e prestação de serviços, riscos que podem comprometer a saúde do homem, dos animais, do meio ambiente ou a qualidade dos trabalhos desenvolvidos ${ }^{2: 4}$.

Outro fator importante está relacionado à legalização da biossegurança no Brasil, que está veiculada à Lei 8.974, de 5 de janeiro de 1995, que criou a Comissão Técnica Nacional de Biossegurança (CTNBio), uma dimensão ampla que extrapola a área da saúde e do trabalho, sendo empregada quando há referência ao meio ambiente e à biotecnologia .

Nos serviços de saúde existem muitas áreas insalubres, de graduações variáveis, dependentes da complexidade e do tipo de atendimento prestado. Nos serviços de emergência, os riscos inerentes à prestação da assistência de enfermagem aumentam consideravelmente 0 índice de infecções, visto que os profissionais e clientes são expostos a um ambiente de trabalho que facilita o surgimento de infecç̃oes cruzadas, pela grande demanda de pacientes politraumatizados, com sangramentos e eliminações de secreções.

Embora o problema das infecções sempre tenha ocorrido, como, por exemplo, as infecções pós-cirúrgicas, transmissão da hepatite $B$, herpes simples e tantas outras, os profissionais de enfermagem, por sua vez, nem sempre estiveram conscientes disso e propensos a seguir os passos necessários para eliminar e diminuir os riscos para os pacientes e para si próprios.

A infecção hospitalar (IH), tanto no Brasil como nos outros países do mundo, representa importante problema de saúde pública ${ }^{3}$. No Brasil, um trabalho publicado em 1995 mostra que o problema da infecção hospitalar já era considerado grave, uma vez que o índice de IH encontrado nos hospitais brasileiros foi de 15,5\%, correspondendo a 1,18 episódio de infecção por paciente internado com IH. Além disso, considera-se mais um agravante a essa situação o fato de as instituições de saúde públicas possuírem a maior taxa de prevalência de IH no país, 18,4\% ${ }^{4}$.

Assim, o conhecimento e a conscientização dos riscos de transmissão de infecções e das limitações dos processos de desinfecção e esterilização são imprescindíveis para que se possam tomar as devidas precauções.

Cabe então ressaltar o conceito de Infecção Hospitalar, segundo a Portaria №. 2616/98 do Ministério da Saúde, como sendo aquela adquirida após admissão do paciente e que se manifesta durante a internação ou após a alta, quando puder ser relacionada com a internação ou procedimentos hospitalares. São também convencionadas infecções hospitalares aquelas manifestadas antes de 72 horas da internação quando associadas a procedimentos diagnósticos e/ou terapêuticos, realizados durante este período ${ }^{5}$.

A maioria das infecções hospitalares manifesta-se como complicações de pacientes gravemente enfermos, decorrentes de um desequilíbrio entre sua flora microbiana normal e seus mecanismos de defesa. Assim, na aquisição das infecções hospitalares, os microorganismos têm um papel passivo, cabendo ao homem o papel ativo; logo, será sobre suas ações o maior enfoque do controle dessas infecções.

Atualmente, as normas consoantes à biossegurança são motivos de preocupação, tanto por parte da Comissão de Controle de Infecção Hospitalar (CClH) quanto pelos Serviços de Medicina Ocupacional. A utilização de precauções básicas auxilia os profissionais nas condutas técnicas adequadas à prestação dos serviços, através do uso correto de equipamento de proteção individual (EPI), de acordo com a Norma Regulamentadora $n^{0} .6$ (NR-6) da Portaria $n^{0}$. 3.214, de 08.06.78. Essas medidas devem gerar melhorias na qualidade da assistência e diminuição de custos e infecções advindos da prática hospitalar tanto para os profissionais como para os clientes e seus familiares $s^{6,7}$.

Dentre as medidas destacam-se os equipamentos de proteção individual (EPIs), que se destinam a proteger os profissionais nas operações de riscos de exposição ou quando houver manipulação de produtos químicos e biológicos, bem como riscos de cortes com materiais perfurocortantes ${ }^{8}$. Os EPls podem ainda ser considerados um dispositivo de uso individual destinado a proteger a integridade física e a saúde do trabalhador? ${ }^{7}$.

A biossegurança, portanto, como fenômeno complexo, não pode ser reduzida a aspectos da lógica racional, devendo ser consideradas as influências de natureza sociocultural. Nessa perspectiva, investigar a biossegurança como fenômeno psicossocial, subsidiado pela teoria das representações sociais, torna-se importante para se conhecerem aspectos subjetivos que influenciam nas condutas e atitudes dos profissionais de enfermagem, especialmente dos serviços de emergência. Esta teoria pode evidenciar imagens e conhecimentos associados diretamente à biossegurança, podendo contribuir para 0 entendimento dos comportamentos e práticas dos profissionais de saúde e suas influências nos índices de infecção hospitalar. 
Dessa forma, este estudo tem como objetivo apreender e analisar as representações sociais da biossegurança elaboradas pelos profissionais de enfermagem do serviço de emergência de um hospital público.

\section{BIOSSEGURANÇA COMO OBJ ETO DE REPRESENTAÇÕES SOCIAIS}

A Teoria das Representações Sociais é uma forma sociológica de Psicologia Social, originada na Europa com a publicação feita por Serge Moscovici no seu estudo La Psychanalyse: Son image et son public ${ }^{9}$. Moscovici é um teórico que se fundamenta no conceito de representação coletiva desenvolvido por Durkheim (1912) e propõe uma nova concepção teórico-conceitual capaz de atender às questões colocadas pelos problemas da modernidade ${ }^{10}$.

A representação social (RS) é definida como uma forma de conhecimento do senso comum. Ela está diretamente relacionada à maneira como as pessoas entendem e introjetam as informações de acordo com os referenciais que possuem; os indivíduos irão reelaborar o saber científico segundo sua própria conveniência, ou seja, de acordo com os meios e recursos que têm ${ }^{11}$.

As representações sociais podem ser também compreendidas como uma forma de conhecimento, socialmente elaborado e partilhado, tendo uma intenção prática e concorrendo à construção de uma realidade comum a um conjunto social ${ }^{12}$. Elas se manifestam em palavras, sentimentos e condutas e se institucionalizam. Portanto, podem e devem ser analisadas a partir da compreensão das estruturas e dos comportamentos sociais. Sua mediação privilegiada, porém, é a linguagem, tomada como forma de conhecimento e de interação social ${ }^{9}$.

A elaboração e o funcionamento de uma representação podem ser compreendidos através dos processos de objetivação e ancoragem. A objetivação e a ancoragem são as formas específicas em que as representações sociais estabelecem mediações, trazendo para um nível quase material a produção simbólica de uma comunidade e dando conta da concreticidade dessas representações na vida socia/ ${ }^{13: 81}$.

Dessa forma, realizar este estudo justifica-se pela importância da temática para a Enfermagem, por serem os profissionais dessa área os que mais tempo permanecem com os pacientes, realizando a maioria dos procedimentos, muitos deles invasivos, ultrapassando as barreiras naturais do organismo. Esses procedimentos, se realizados sem os cuidados, ou sem as medidas de biossegurança necessárias para evitar uma infecção cruzada, podem trazer sérias complicações, resultando no aumento da permanência hospitalar, custo com a internação e, especialmente, muito sofrimento e insegurança para o paciente.

Refletindo-se sobre o homem como um ser sócio-histórico em sua relação com a natureza, suas ações e atitudes estão constantemente permeadas pelas relações sociais estabelecidas em determinado contexto. Assim, observou-se que os pacientes e os profissionais de saúde estão constantemente expostos a um grande risco de infecção cruzada.

Face ao exposto, propomo-nos a estudar as representações sociais que orientam as condutas dos profissionais de
Enfermagem relacionadas à biossegurança. Espera-se que a compreensão de tais representações possa fornecer subsídios para elaboração de novas estratégias de enfrentamento dos problemas decorrentes das práticas dos enfermeiros que ainda não incorporaram as medidas de biossegurança.

\section{METODOLOGIA}

Trata-se de uma pesquisa exploratória desenvolvida no serviço de emergência de um hospital público de grande porte, localizado em Teresina, com profissionais de enfermagem.

A amostra foi definida partindo da concepção de saturação de idéias e da disponibilidade dos profissionais em participarem desse estudo, que, após aceitação verbal, culminou na assinatura do termo de consentimento livre e esclarecido, após aprovação do Comitê de Ética e Pesquisa - CEP/HGV, de acordo com a Resolução 196/96 do Conselho Nacional de Saúde ${ }^{14}$.

As variáveis gênero, faixa etária e categoria profissional foram responsáveis pelo perfil dos sujeitos da amostra, os quais compreenderam 60 profissionais de enfermagem, dos quais 4 se encontravam na faixa etária de 20 a 30 anos; 22, na faixa etária 31 a 40; e 34, na faixa etária 41 anos e mais. Quanto ao sexo, 6 eram do sexo masculino e 54, do feminino. Outro fator que se procurou observar está relacionado à categoria profissional: 10 tinham nível superior e 50, nível médio.

Os dados foram produzidos a partir do teste de associação livre de palavras, com as palavras-estímulos: biossegurança e infecção hospitalar. 0 teste de associação livre de palavras, originalmente desenvolvido por Jung na prática clínica e adaptado no campo da psicologia social por Di Giacomo, vem sendo amplamente utilizado nas pesquisas sobre representações sociais ${ }^{15}$.

Uma vez organizados os dicionários e o banco de dados, os resultados do teste de associação livre de palavras foram processados no software Tri-Deux Mots, indicado para tratamento de questões abertas e fechadas, e interpretados por meio da Análise Fatorial de Correspondência (AFC). 0 mesmo permite verificar correlações entre grupos, assim como visualizar as relações de atração e afastamento entre os elementos do campo representacional a propósito de determinado objeto.

Desse modo, configuraram-se graficamente as representações sociais relativas aos estímulos indutores, revelando-se aproximações e oposições das modalidades na construção dos fatores analisadas através da AFC, definindo, assim, a objetivação da biossegurança.

\section{CONSTRUINDO AS REPRESENTAÇÕES SOCIAIS SOBRE A BIOSSEGURANÇA}

A Teoria das Representações Sociais aplicada na pesquisa sobre o fenômeno da biossegurança possibilita edificação de conceitos, explicações e afirmações que se originam no cotidiano através das comunicações interindividuais e do conhecimento socialmente elaborado e compartilhado. Pautada nessa abordagem, explicitada pela teoria, a realização deste estudo teve como elemento determinante as evocações dos 
profissionais de enfermagem que vivenciam problemas relacionados à prática de enfermagem no serviço de emergência, sobre a biossegurança.

Destaca-se, assim, que a Teoria das Representações Sociais, capaz de identificar vários aspectos psicossociológicos envolvidos na biossegurança, considera a vivência dos profissionais no trabalho enquanto fenômenos de produção e organização de conhecimentos de sujeitos sociais particulares, revelando-se em um excelente instrumento no diagnóstico psicossocial que possibilitou o entendimento da biossegurança na interface dos condicionantes psicológicos, sócio-culturais e psicossociais.

Os dados obtidos a partir do Teste de Associação Livre de Palavras e processados pelo software Tri-Deux Mots deram origem a um conjunto de 2.120 palavras como respostas para os estímulos infecção hospitalar e biossegurança. Destas, 95 com significados diferentes. Esta redução deu-se pela formação dos dicionários, através do agrupamento pela similaridade semântica existente entre as palavras. Por exemplo, expressões como troca de lençóis e roupa de cama foram reduzidos a uma única palavra, lençol, para se referir à biossegurança.

Através do Gráfico 1, pode-se observar o conteúdo das representações sobre biossegurança, tornando-se possível, a partir da objetivação configurada no campo representacional, fazer a leitura e interpretação das modalidades de opinião pelas palavras evocadas, correlacionando-as com as variáveis fixas (sexo, faixa etária e categoria profissional) organizadas segundo os eixos ou fatores (F1 e F2).

No Gráfico 1, disposto a seguir, encontram-se configurados ou representados dois lados fatoriais $\mathrm{F} 1$ e F2, bem como as variáveis fixas que contribuíram para a formação dos dois eixos.

\section{Gráfico 1:}

Representação gráfica do plano fatorial sobre biossegurança.

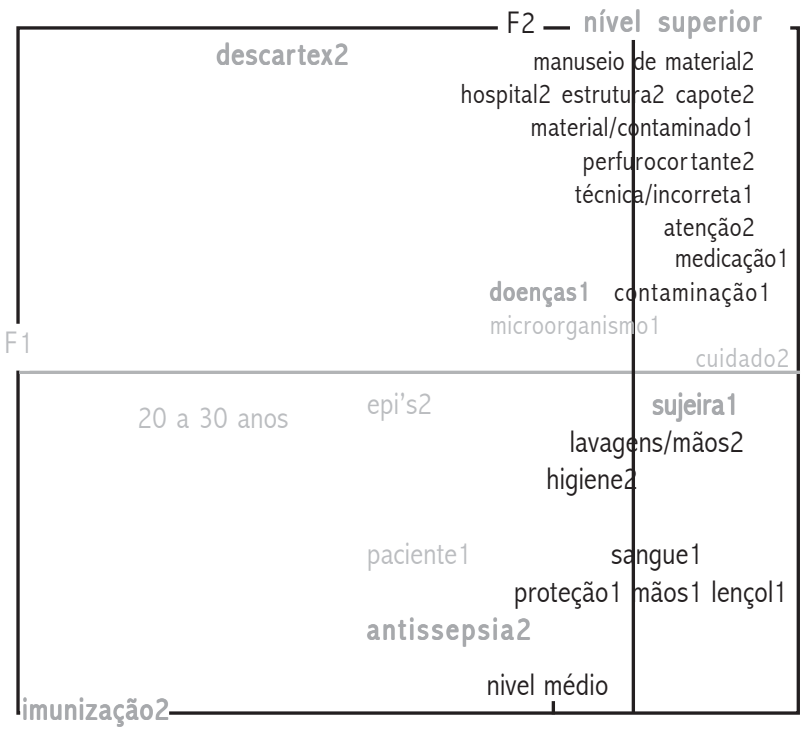

\begin{tabular}{|c|c|c|}
\hline Plano Fatorial & Palavras Indutores & Varáveis Fixas \\
\hline $\begin{array}{l}\text { Fator } 1(F-)=\text { [eixo horizontal - esquerda } \\
\text { (negativo) e direita (positivo)] } \\
\text { Fator } 2 \text { (F2) = [eixo vertical - superior } \\
\text { (positivo) e inferior (negativo)] } \\
\text { Cinza escuro - modalidades que existem } \\
\text { em ambos os vetores }\end{array}$ & $\begin{array}{l}1 \text { - Infecção Hospitalar } \\
2 \text { - Biossegurança }\end{array}$ & $\begin{array}{l}15 \text { - Sexo } \\
16 \text { - Idade } \\
17 \text { - Categoria } \\
\text { Funcional }\end{array}$ \\
\hline
\end{tabular}

Durante o processo analítico das evocações, foram consideradas as correlações existentes entre os diferentes grupos de palavras, identificando assim as aproximações e oposições das modalidades.

Os dados apresentados, a partir do Gráfico 1, começam pelas palavras que se encontram no lado esquerdo (negativo) e direito (positivo) do F1 (eixo horizontal), seguidas pelas palavras que se encontram no lado inferior (negativo) e lado superior (positivo) do F2 (eixo vertical).

\section{Fator 1 - lado esquerdo (negativo)}

Destacam-se no gráfico do lado esquerdo (negativo) do fator 1 (eixo horizontal) as representações sociais da infecção hospitalar e biossegurança, elaboradas por profissionais de enfermagem do nível médio, de 20 a 30 anos de idade.

Segundo esse grupo de sujeitos, infecção hospitalar é sinonímia de doença e paciente. Através dessas modalidades, os profissionais de enfermagem reconhecem que a variedade de doenças agrupadas no mesmo local e a quantidade exacerbada de pacientes no serviço de emergência favorecem o surgimento e disseminação da infecção hospitalar.

A representação da biossegurança é descrita pelos profissionais de enfermagem como: EPIs (equipamentos de proteção individual), anti-sepsia e descartex. Essas representações apresentam-se fortemente vinculadas aos aspectos benéficos da biossegurança, por proporcionar medidas de proteção individual e coletiva para os pacientes e profissionais no serviço de emergência.

\section{Fator 1 - lado direito (positivo)}

No lado oposto (direito positivo) do mesmo fator (F1), observase a delimitação do agrupamento das representações sociais sobre os mesmos estímulos infecção hospitalar e biossegurança, constituídas por profissionais de enfermagem do nível superior.

Neste lado do eixo, infecção hospitalar é sinonímia de sujeira. Essa palavra surgiu na elaboração do dicionário, que se deu através do agrupamento de palavras com similaridades semânticas destacadas entre as evocações: falta de higiene e falta de limpeza. Através dessas modalidades, os profissionais de enfermagem reconhecem que a infecção hospitalar é favorecida pelo descuido dos pacientes com relação à sua higiene pessoal bem como pela limpeza inadequada do ambiente hospitalar em que estes se encontram.

0 termo limpeza hospitalar vem sendo com freqüência considerado sinônimo de higiene hospitalar ou zeladoria hospitalar. Tratando-se de uma definição específica de limpeza, podemos entendê-la como o processo de remoção de sujidades. Consideramos então limpeza hospitalar a limpeza das superfícies fixas e equipamentos permanentes das diversas áreas hospitalares, o que inclui pisos, paredes, janelas, mobiliários, equipamentos e instalações sanitárias ${ }^{16}$.

A biossegurança é expressa pelos profissionais, nesse fator, no lado direito (positivo), como cuidado. Dessa forma, o cuidado pode ser avaliado, pelos sujeitos sociais, como fator essencial para a prevenção, minimização ou eliminação de riscos inerentes às atividades de pesquisa, produção, ensino, desenvolvimento tecnológico e prestação de serviços ${ }^{17}$. 


\section{Fator 2 - lado inferior (negativo)}

No fator 2 (eixo vertical), no lado inferior negativo do Gráfico 1, destacam-se as representações sobre infecção hospitalar e biossegurança elaboradas por profissionais na faixa etária de 20 a 30 anos, da categoria nível médio.

As modalidades correspondentes às variáveis de opinião evocadas por esses profissionais em resposta ao estímulo infecção hospitalar são representadas pelas palavras: sangue, mãos, sujeira, lençol e proteção. Desse modo, em relação a esse estímulo indutor, configuram-se evocações contraditórias, visto que, ao tempo em que os profissionais referem modalidades que favorecem as infecções cruzadas, como sangue, mãos, sujeira e lençol, associam proteção como forma de evitar essas infecções.

Em relação à biossegurança, os profissionais destacam as palavras: lavagem das mãos, higiene, anti-sepsia e imunização, sendo que essas duas últimas estão presentes em ambos os vetores. A biossegurança revela-se favorável em todas as manifestações (evocações), nas quais os profissionais demonstram conhecer as normas consoantes à biossegurança e a importância dessas medidas para a qualidade da assistência e diminuição de infecções advindas da prática hospitalar.

A higienização das mãos, nos programas de prevenção e controle das infecções hospitalares, é uma prática prioritária, considerando ser a ação isoladamente mais importante para reduzir as taxas dessas infecções no ambiente hospitalar. 0 objetivo principal do processo de higienização das mãos é o de reduzir a transmissão de microorganismos pelas mãos, prevenindo as infecções ${ }^{18}$.

A diminuição dos microorganismos da pele e mucosa pode se processar em diferentes níveis dependendo dos processos empregados de limpeza ou anti-sepsia. 0 procedimento de limpeza remove a microbiota transitória humana através da técnica de lavagem simples das mãos ${ }^{19}$.

No ambiente hospitalar há diversas infecções preveníveis por imunização, que podem ser disseminadas entre pacientes, entre profissionais da área da saúde ou entre ambos os grupos. Dessa forma, os benefícios da imunização incluem a proteção aos profissionais e pacientes, a interrupção da disseminação de doenças infecciosas e de surtos intra-hospitalares, além da proteção indireta de pessoas não vacinadas contra algumas doenças ${ }^{20}$.

\section{Fator 2 - lado superior (positivo)}

No fator 2 (eixo vertical), no lado superior positivo do Gráfico 1, encontram-se as objetivações de infeç̧ão hospitalar e biossegurança, construídas por profissionais do nível superior.

No que se refere à palavra-estímulo infecção hospitalar, os sujeitos pesquisados contribuíram com a objetivação nesse fator com as seguintes modalidades: material contaminado, técnica incorreta, medicação, contaminação e doenças. A partir dessas evocações, os profissionais de enfermagem evidenciam que os fatores que contribuem para infecção hospitalar estão relacionados não somente com 0 ambiente hospitalar, mas também com a falta de capacitação e/ ou qualificação dos profissionais que atuam nesse serviço, como evidenciado nas evocações: técnica incorreta e medicação.
Ainda com relação ao mesmo fator (F2 - lado superior), os profissionais apresentam como sinonímia de biossegurança: manuseio de material, estrutura, capote, descartex, perfurocortante e atenção. Em consonância com análises feitas até então sobre as palavras evocadas em atendimento ao estímulo biossegurança, da mesma forma, nesse lado do fator F2, é representado por palavras que reforçam a proteção oferecida pelas medidas de biossegurança, tanto individuais (uso de capote, atenção, manuseio adequado de perfurocortantes), quanto àquelas relacionadas ao ambiente (estrutura adequada, descartex).

Os acidentes com materiais perfurocortantes são o principal risco relacionado aos resíduos de serviço de saúde (RSS), e a forma de evitá-los é adotar o uso de coletores de resíduos sólidos rígidos adequados, como o descartex.

Dessa forma, os dados apresentados dizem respeito à análise de 26 variáveis de opinião que foram apreendidas na representação gráfica com maior freqüência de contribuição para os dois eixos. Nelas pode-se observar a contribuição de cada modalidade na formação dos fatores a partir da freqüência com que cada uma se apresenta. Assim, pode-se observar a distribuição das representações nos dois fatores (F1 e F2). Os valores numéricos da contribuição fatorial em resposta ao estímulo infecção hospitalar foi no total de 705 e biossegurança, no total de 1.189 .

Observa-se que, na análise fatorial do estudo, algumas palavras tiveram contribuições significativas nos dois fatores (F1 eF2). Como resposta ao estímulo infecção hospitalar, as palavras doença e sujeira foram as modalidades evocadas que contribuíram com os dois fatores e, como resposta ao estímulo biossegurança, as palavras descartex, anti-sepsia e imunização foram as modalidades evocadas que também contribuiram com os dois fatores. As palavras com maior freqüência, resultantes dos dois estímulos e que contribuíram com os dois fatores, foram: descartex, anti-sepsia e imunização.

Dessa forma, a combinação dos dois critérios, maior freqüência de evocações e contribuição nos dois fatores, resultaram nas palavras: doença, sujeira, descartex, antisepsia e imunização como evocações mais significativas pertencentes às representaç̃̃es sociais dos sujeitos da pesquisa sobre biossegurança no serviço de emergência.

As representações sociais, enquanto conhecimento do senso comum sobre determinado objeto ou problemas sociais específicos e importantes na vida dos grupos, alimentam e são produzidas numa interdependência do sujeito/objeto/interação social. Os fenômenos perceptivos, imagens, as opiniões, crenças ou atitudes que formam o tecido atômico das representações sociais somente adquirem estruturação lógica no entrelaçamento dos vínculos entre estes elementos, o que possibilita a atribuição de significação aos processos psicossociais ${ }^{13}$.

Assim, é possível apreender os aspectos psicossociais, presentes nas culturas, nas manifestações, nos posicionamentos, nos sentimentos e nas evocações desses sujeitos, como componentes sociais determinantes das medidas de biossegurança, visto que contribuem para orientação de condutas e construção do conhecimento em relação ao fenômeno social do estudo. 


\section{CONSIDERAÇÕES FINAIS}

A biossegurança como objeto social constituiu o foco deste estudo, a partir do qual os profissionais de enfermagem puderam explicitar as representações sociais através do instrumento de pesquisa, o Teste de Associação Livre de Palavras.

0 posicionamento diante da biossegurança é evidenciado com elementos favoráveis, tais como: descartex, anti-sepsia e imunização. Isso mostra que a ênfase dada pelos profissionais ao reconhecimento do valor da proteção e prevenção responde aos apelos do seu grupo social e corrobora a proposta de um programa de controle de infecção hospitalar e biossegurança, tal com definido atualmente no âmbito da Enfermagem.

As concepções sobre o estímulo infecção hospitalar foram objetivadas através das evocações: doença e sujeira, como conseqüência da prática hospitalar, influenciando implicitamente na transmissão de infecção em todas as ações de enfermagem no serviço de emergência.

Desse modo, percebe-se através das representações um posicionamento de contradições, visto que os profissionais, ao mesmo tempo em que referem modalidades de conteúdo

\section{Referências}

1. Mastroeni MF. Introdução à biosseguranca. In: Biosseguranca aplicada a laboratório e serviços de saúde. São Paulo (SP): Atheneu; 2006.

2. Hinrichsen SL. Lei de Biossegurança Nacional: alguns aspectos importantes. In: Biosseguranca e controle de infecções: risco sanitário hospitalar. Rio de Janeiro (RJ): MEDSI; 2004

3. Lacerda RA. Infecção hospitalar e sua relação com a evolução das práticas de assistência em saúde. In: ___ C Controle de infecção em centro cirúrgico: fatos, mitos e controvérsia. São Paulo (SP): Atheneu; 2003.

4. Prade SS, Oliveira ST, Rodrigues R, Nunes FA, Félix JK, Pereira M. Estudo brasileiro da magnitude das infecções hospitalares em hospitais terciários. Rev Control Infec Hosp 1995; 2 (2): 4-8.

5 Ministério da Saúde (BR). Portaria $n^{0}$ 2616, de 12 de maio de 1998. Estabelece diretrizes e normas para a prevenção e controle das infecções hospitalares. Brasília (DF); 1998.

6. Santos Junior AF, Schindler Junior AFR, Pinto AM. Emprego do planosequência no gerenciamento da roupa hospitalar: Avaliação de aspectos de biossegurança. Rev Prat Hosp 2005 mar/abr; 7(38).

7. Skraba I, Nickel R, Wotkoski SR. Barreiras de contenção: EPI e EPCs. In: Mastroeni MF. Biosseguranca aplicada a laboratório e serviços de saúde. São Paulo(SP): Atheneu; 2006.

8. Marudian LBA. Equipamento de proteção individual. In: Hirata MH, Mancini Filho J. Manual de Biosseguranca. São Paulo(SP): Manole; 2002

9. Farr RM. Representações sociais: a teoria e sua história. In: Guareschi PA, Jovchelovitch S, organizadoras. Textos em representações sociais. Petrópolis(RJ): Vozes; 2003.

10. Nóbrega SM. Sobre a teoria das representações sociais. In: Moreira ASP. Representações sociais: teoria e prática. João Pessoa (PB): Ed Universitária UFPB; 2001. favorável, ao reconhecerem a importância e o valor das normas de biossegurança, em contrapartida absorvem, apenas parcialmente, o que determina o programa de Prevenção e Controle da Infecção Hospitalar relacionado com a biossegurança.

À medida que são objetivadas as Representações Sociais da biossegurança, ancoradas nos aspectos ideológicos, históricos, sociais e sócio-culturais, evidencia-se um conflito entre as propostas dos programas de incentivo às práticas de biossegurança que focalizam predominantemente os aspectos epidemiológicos, biológicos, econômicos, e quase nunca o ponto de vista social e psicológico. Nesse sentido, para se falar em políticas de controle de infecção hospitalar, devem-se considerar os determinantes das práticas de biossegurança que se encontram atreladas às crenças, valores, normas sociais, dentre outros.

Neste estudo, podemos considerar que a biossegurança não foi ainda incorporada como um conjunto de medidas necessárias ao controle da infecção hospitalar, mas representada de forma fragmentada conforme se mostra nos conceitos, valores, preconceitos e imagens, na medida em que predomina apenas parte das normas de proteção individual e coletiva, ficando de fora todas as outras medidas necessárias e fundamentais à quebra da cadeia de infecção.

11. Moscovici S. A representação social da psicanálise. Rio de Janeiro(RJ): Zahar; 1978.

12. Jodelet D. As representações sociais. Rio de Janeiro(RJ): Ed UERJ; 2001.

13. Jovchelovitch S. Vivendo a vida com os outros: intersubjetividade, espaço público e representações sociais. In: Guareschi PA, Jovchelovitch $\mathrm{S}$, organizadoras. Textos em representações sociais. Petrópolis (RJ): Vozes; 2003.

14. Ministério da Saúde (BR). Conselho Nacional de Saúde. Diretrizes e normas reguladoras de pesquisa envolvendo seres humanos. Resolução n 196 de 10 de outubro de 1996. Inf Epidemiol SUS 1996; 5 (2): 14-41.

15. Nóbrega SMN, Coutinho MPL. 0 texte de associação livre de palavras. In: Coutinho MPL. Representações sociais: abordagem interdisciplinar. João Pessoa (PB): Universitária UFPB; 2003.

16. Yamaushi NI, Lacerda RA, Gabrielloni MC. Limpeza hospitalar. In: Fernandes AT, Fernandes MOV, Ribeiro Filho N. Infecção hospitalar e suas interfaces na área da saúde. São Paulo (SP); 2000.

17. Teixeira P, Valle S. Biossegurança: uma abordagem multidisciplinar. Rio de Janeiro (RJ): FIOCRUZ; 1996.

18. Lira MC, Hinrichsen SL, Anjos AB, Borges MGA, Pereira MGB, Danda GJN. Higienização das mãos. In: Biosseguranca e controle de infecções: risco sanitário hospitalar. Rio de Janeiro (RJ): MEDSI; 2004 19. Graziano KU, Silvia A, Bianchi ERF. Limpeza, desinfecção, esterilização de artigos e anti-sepsia. In: Fernandes AT, Fernandes MOV, Ribeiro Filho N. Infecção hospitalar e suas interfaces na área da saúde. São Paulo (SP): Atheneu; 2000.

20. Conceição FM, Cavalcante NJF, Ayub MA. Imunização em profissionais de saúde. In: Fernandes AT, Fernandes MOV, Ribeiro Filho N. Infecção hospitalar e suas interfaces na área da saúde. São Paulo (SP) São Paulo (SP): Atheneu; 2000. 